\title{
Aberrant integrin $\alpha v$ and $\alpha 5$ expression in prostate adenocarcinomas and bone-metastases is consistent with a bone-colonizing phenotype
}

\author{
Brendan Connell ${ }^{1,2}$, Pavel Kopach ${ }^{3,4}$, Wenying Ren $^{1}$, Raghav Joshi ${ }^{1}$, Stephen Naber ${ }^{3}$, Ming Zhou ${ }^{3}$, \\ Paul Mathew ${ }^{1}$
}

${ }^{1}$ Division of Hematology-Oncology, Tufts Medical Center, Boston, MA, USA; ${ }^{2}$ Department of Hematology-Oncology, Lahey Hospital \& Medical Center, Burlington, MA, USA; ${ }^{3}$ Department of Pathology, Tufts Medical Center, Boston, MA, USA; ${ }^{4}$ Department of Pathology, Memorial SloanKettering Cancer Center, New York, NY, USA

Contributions: (I) Conception and design: P Mathew, B Connell; (II) Administrative support: B Connell; (III) Provision of study materials or patients: S Naber, P Mathew; (IV) Collection and assembly of data: B Connell, P Kopach, W Ren, R Joshi; (V) Data analysis and interpretation: All authors; (VI) Manuscript writing: All authors; (VII) Final approval of manuscript: All authors.

Correspondence to: Paul Mathew, MD. Division of Hematology-Oncology, Tufts Medical Center, 800 Washington St., Boston, MA 02111, USA. Email: pmathew@tuftsmedicalcenter.org.

Background: Collaborative signaling between fibronectin-binding $\alpha \mathrm{v}$ and $\alpha 5$ integrins has been implicated in the lethal dissemination of prostate cancer in the bone-metastatic niche, the major source of morbidity and mortality in the disease.

Methods: We assessed the frequency and pattern of expression of these integrins in primary high-grade adenocarcinomas and bone metastases compared to the physiological gland. Formalin-fixed paraffinembedded (FFPE) radical prostatectomy (RP) samples $(n=25)$ containing $\geq$ Gleason grade 4 cancer and decalcified surgical or diagnostic bone metastatic samples from 10 patients were stained for integrin $\alpha \mathrm{v}$ (ITGAV) and integrin $\alpha 5$ (ITGA5) expression. Antibody optimization and antigen-retrieval was performed beforehand.

Results: ITGAV was exclusively expressed in the basal layer of physiological prostate glands whereas $\alpha \mathrm{v}$ expression was invariably recapitulated in the malignant gland and bone metastases (100\%) in multiple distinct patterns: epithelial membranous, basilar/luminal membranous, punctate cytoplasmic, intense foci as single cells or clusters, and rim stromal layers. The luminal/basilar layer of ITGAV expression was striking in cribriform carcinomas, suggestive of a role in molecular pathogenesis. ITGA5 infrequently highlighted the basal layer of the physiological gland, was absent in primary adenocarcinoma, but was expressed with ITGAV exclusively in bone metastases (71\%).

Conclusions: We conclude that ITGAV expression is aberrantly expressed in high frequency in high-grade prostatic adenocarcinomas in patterns suggestive of recapitulated basal cell functions, consistent with a stemregulatory role that has been proposed. Co-expression and enrichment of $\alpha \mathrm{v}$ and $\alpha 5$ in osseous metastases supports their proposed collaborative role in colonization of the bone microenvironment and as candidate targets for therapy.

Keywords: Bone metastases; cribriform carcinoma; integrins; prostate cancer

Submitted Nov 19, 2019. Accepted for publication Jun 30, 2020.

doi: $10.21037 /$ tau-19-763

View this article at: http://dx.doi.org/10.21037/tau-19-763 


\section{Introduction}

Prostate cancer disseminates within the hematopoietic bone marrow in the proximal appendicular and axial skeleton in the large majority of men with advanced disease. Morbidity and mortality from prostate cancer is closely attributed to the burden of disease in bone, referred to as the lethal phenotype of prostate cancer (1). Identification of the molecular mechanisms that underpin the efficient colonization of the bone marrow by prostate cancer cells are essential to define novel molecular therapeutic strategies in the disease.

Human bone marrow-derived mesenchymal stromal cells (hBM-MSCs) have been implicated as architects of the hematopoietic niche $(2,3)$ which is in turn subverted by tumor cells (4) that have disseminated to the bone microenvironment. Based on the hypothesis that hBM-MSCs specify a potent molecular niche for human prostate cancer cells, the mechanisms that drive chemotaxis and adhesionmediated survival of prostate cancer cells co-cultured with hBM-MSCs was identified. Specifically, proteolytic fragments of fibronectin secreted by hBM-MSCs signaling via fibronectin-binding integrins $\alpha 5 \beta 1(5)$ and $\alpha \mathrm{v}(6,7)$ was proposed as a candidate seed-soil mechanism to explain the strong tropism of prostate cancer cells to the bone marrow microenvironment. To qualify this molecular hypothesis with pathological expression of these integrins and to further inform translational therapeutics that simultaneously target these cross-regulatory integrins (7), the patterns of expression of integrin $\alpha 5$ (ITGA5) and integrin $\alpha \mathrm{v}$ (ITGAV) in primary prostatic adenocarcinomas and bone metastases were identified. We present the following article in accordance with the STROBE reporting checklist (available at http:// dx. doi. org/10. 21037/tau-19-763).

\section{Methods}

All procedures performed in this study were in accordance with the Declaration of Helsinki (as revised in 2013) and approved by Tufts Medical Center Institutional Review Board (reference number 11987). Because of the retrospective nature of the research, the requirement for informed consent was waived.

\section{Antibody optimization}

Cell pellets containing human prostate cancer cell lines PC3, LNCaP, DU-145, and VCAP were formed with Histogel
(Thermo Fisher Scientific, USA). These were fixed in $10 \%$ formalin. Antibodies were selected against ITGA5 (SC-376199, Santa Cruz Biotechnology, Santa Cruz, CA, USA) and ITGAV (EPR16800, Abcam, Cambridge, MA, USA). We performed serial dilutions of the antibodies to identify optimal conditions to distinguish membrane from cytoplasmic staining.

\section{Case selection}

We retrospectively evaluated pathology reports for 231 subjects who had undergone prostatectomy at Tufts Medical Center between 2012-2017. This modern cohort was selected to minimize time-dependent surface antigen degradation within the samples. From the Tufts Medical Center Tissue Biorepository, we selected a study set of 25 cases reported to contain high-grade ( $\geq$ Gleason grade 4 ) prostate adenocarcinoma. We additionally retrieved ten cases of bone metastases from prostate adenocarcinoma, either surgical specimens from hip or spine or bone-marrow biopsies from the posterior-superior iliac crest. Seven of these contained sufficient evaluable tumor.

\section{Tissue processing}

All biorepository prostate samples had been formalinfixed and paraffin-embedded (FFPE), and stored at room temperature. The bone samples had additionally been decalcified [surgical specimens with hydrochloric acid (Decal II) and bone marrow biopsies with formic acid (Immunocal)]. Thin sections were placed on adhesive slides (Newcomer Supply TruBond 380).

\section{Immunobistochemistry}

The samples underwent a citrate antigen retrieval process in a steamer for 20 minutes, then treated with hydrogen peroxide $\left(\mathrm{H}_{2} \mathrm{O}_{2}\right)$ for 10 minutes followed by a goat serum blocking solution for 10 minutes. The slides were incubated with the primary antibodies (ITGA5 at 1:1,000 and ITGAV at $1: 2,000)$ for $60 \mathrm{~min}$ at room temperature. The secondary antibody was a polymer anti-mouse antibody for ITGA5 and anti-rabbit antibody for ITGAV, which were incubated at room temperature for 30 minutes. The chromagen was 3,3'-diaminobenzidine (DAB), incubated for 6 minutes. Slides were counterstained with hematoxylin, treated with a bluing solution (4\% ammonium hydroxide aqueous) and dehydrated to xylene for coverslipping. 

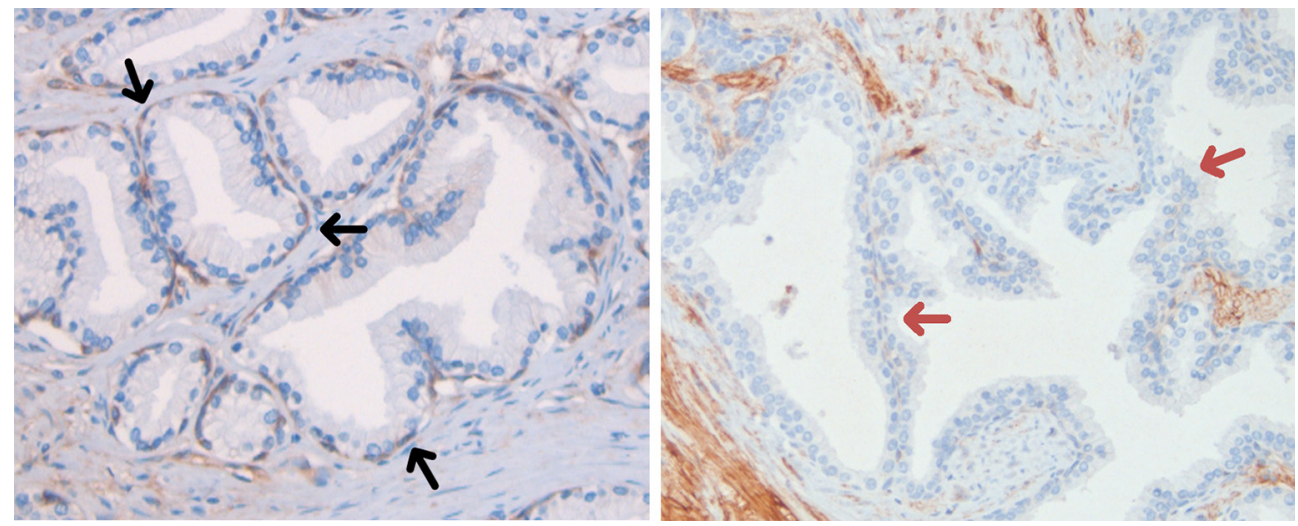

Figure 1 Benign prostate glands with ITGAV staining (black arrows, left panel, 40x) and ITGA5 negative staining (red arrows, right panel, $40 \times$ ) in the basal cell layer. ITGAV, integrin $\alpha \mathrm{v}$; ITGA5, integrin $\alpha 5$.

\section{Scoring}

Slides were observed under a light microscope by a pathologist. Quantity, intensity and localization of immunoreactivity were assessed for each slide examining the benign prostate glands, malignant glands, and the adjacent stroma. A semi-quantitative analysis was performed. As previously described for HER2 staining in breast cancer (8), a slide was scored as positive if $\geq 10 \%$ of cells contained at least moderate (2+) staining intensity. Descriptive statistics were used for proportion of tumors expressing patterns of interest.

\section{Results}

\section{Pathologic characteristics of samples}

Twenty-five radical prostatectomy (RP) samples and seven of ten bone metastases containing evaluable tumor were assessed. Of the RP samples, two were scored Gleason score $3+3(8 \%)$, six were $3+4(24 \%)$, nine were $4+3(36 \%)$, five were $4+4(20 \%)$, two were $4+5(8 \%)$, and one was $5+4(4 \%)$. Cribriforming glands were seen in 20 samples (87\%).

\section{Integrin expression in benign glands}

In the basal layer of benign prostate glands, ITGAV was expressed in all 25 samples $(100 \%)$ while ITGA5 was expressed in only 1 of 10 samples examined (10\%). ITGAV and ITGA5 were both negative in the benign luminal layer in all samples (0\%) (Figure 1).

\section{Integrin expression in malignant glands}

In the malignant prostatic epithelium, all 25 samples (100\%) expressed ITGAV but none of the 10 samples expressed ITGA5 (0\%).

\section{Distinct patterns of expression of ITGAV in malignant glands}

After a first round of analysis, we observed and defined distinct patterns of ITGAV expression that recurred in malignant glands that were not mutually exclusive (Figure 2). Of the 25 samples, we noted membranous expression in 24 samples (96\%), punctate cytoplasmic expression in 14 (56\%), basilar/luminal membranous expression in $6(24 \%)$, a rim effect in a thin stromal cell layer around glands or fused glands in $14(56 \%)$, and intense foci of membranous staining in single cells or small clusters of cells in 10 samples $(40 \%)$. The luminal membranous and rim effect were demonstrated in cribriform carcinoma. All patterns of ITGAV expression are tabulated in Table 1.

\section{Integrin expression in bone metastases}

Of the 7 bone metastasis samples analyzed, 5 tumors (71.4\%) expressed ITGA5 and all 7 (100\%) expressed ITGAV (Figure 3). These included recapitulations of the ITGAV patterns seen in primary tumors specifically the membranous, punctate cytoplasmic and intense foci (Figure 3). 

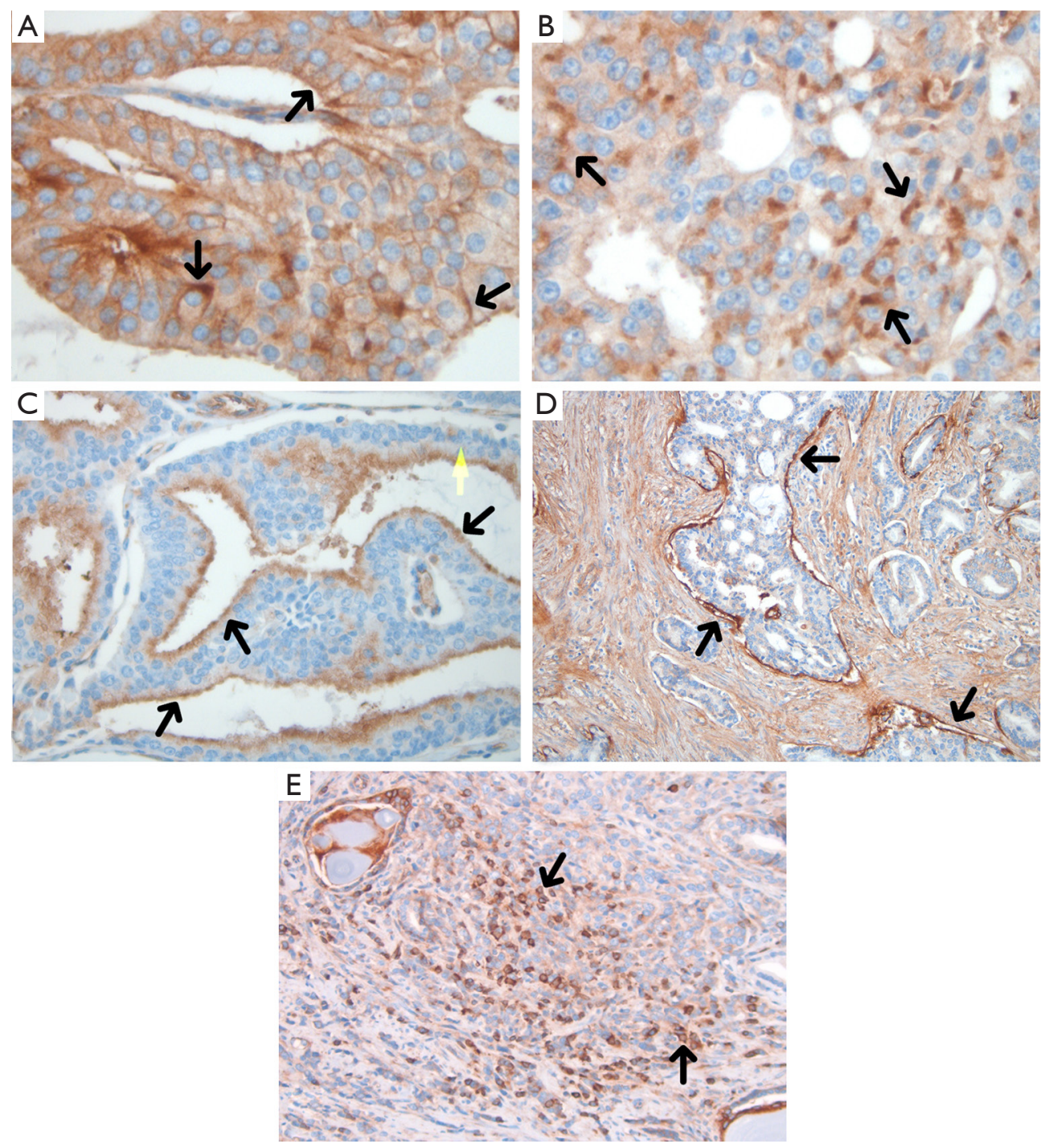

Figure 2 Malignant prostatic epithelium showing distinct patterns of $\alpha$ v expression (black arrows, 20/40×): membranous (A), punctate cytoplasmic (B), basilar/luminal membranous (C), rim stromal (D), and intense foci (E).

\section{Integrin expression in the tumor microenvironment of prostate and bone}

ITGA5 showed strong expression in both the benign and tumor-adjacent stroma. In specific cases, ITGAV showed an enriched intensity in the malignant stroma. ITGAV and ITGA5 expression in capillary endothelium were highlighted in Gleason 5 pattern adenocarcinoma. Osteoclasts stained strongly for ITGAV and endosteal layer cells for ITGA5 which served as positive internal controls. As with high-grade primary tumors, both integrins were also expressed in capillary endothelium in bone (Figure 4).

\section{Discussion}

This study has linked experimental data that implicated ITGA5 and ITGAV in the colonization of the bone microenvironment by prostate cancer cells to primary pathological data in human specimens. There are several key findings to be integrated from the collective pathological observations in prostate and bone that have implications for translational clinical studies. We 
Table 1 Frequency of patterns of integrin $\alpha \mathrm{v}$ expression in prostate gland and bone metastases

\begin{tabular}{lc}
\hline Pattern of expression & ITGAV expression, $\mathrm{n}[\%]$ \\
\hline Benign prostate glands & $25[100]$ \\
Basal epithelium & $0[0]$ \\
Luminal epithelium & \\
Malignant prostate glands & $25[100]$ \\
Any positive staining & $24[96]$ \\
Membranous & $14[56]$ \\
Punctate cytoplasmic & $6[24]$ \\
Luminal membranous & $14[56]$ \\
Rim effect & $10[40]$ \\
Intense foci & \\
Metastatic tumor & $7[100]$ \\
Bone metastases & \\
\hline
\end{tabular}

ITGAV, integrin $\alpha$ V. summarize and discuss these several observations in the following narrative.

\section{Transition of integrin expression from the physiological basal layer to patterned expression in carcinoma}

Expression of the ITGAV was universally expressed and strictly confined to the basal layer of benign glandular epithelium in the prostate. While ITGA5 is infrequently expressed in basal cells, neither integrin is present in luminal cells of benign glands. The basal layer of the prostate gland is essential to anchor the gland to the basement membrane to regulate interactions with stroma and in the generation of a differentiated luminal layer with secretory function. Basal layer cells have both stem-like self-renewal and differentiation capacity. Loss of the basal layer is a hallmark pathological feature in the progression to invasive adenocarcinoma. All primary tumors examined expressed ITGAV in diverse patterns.
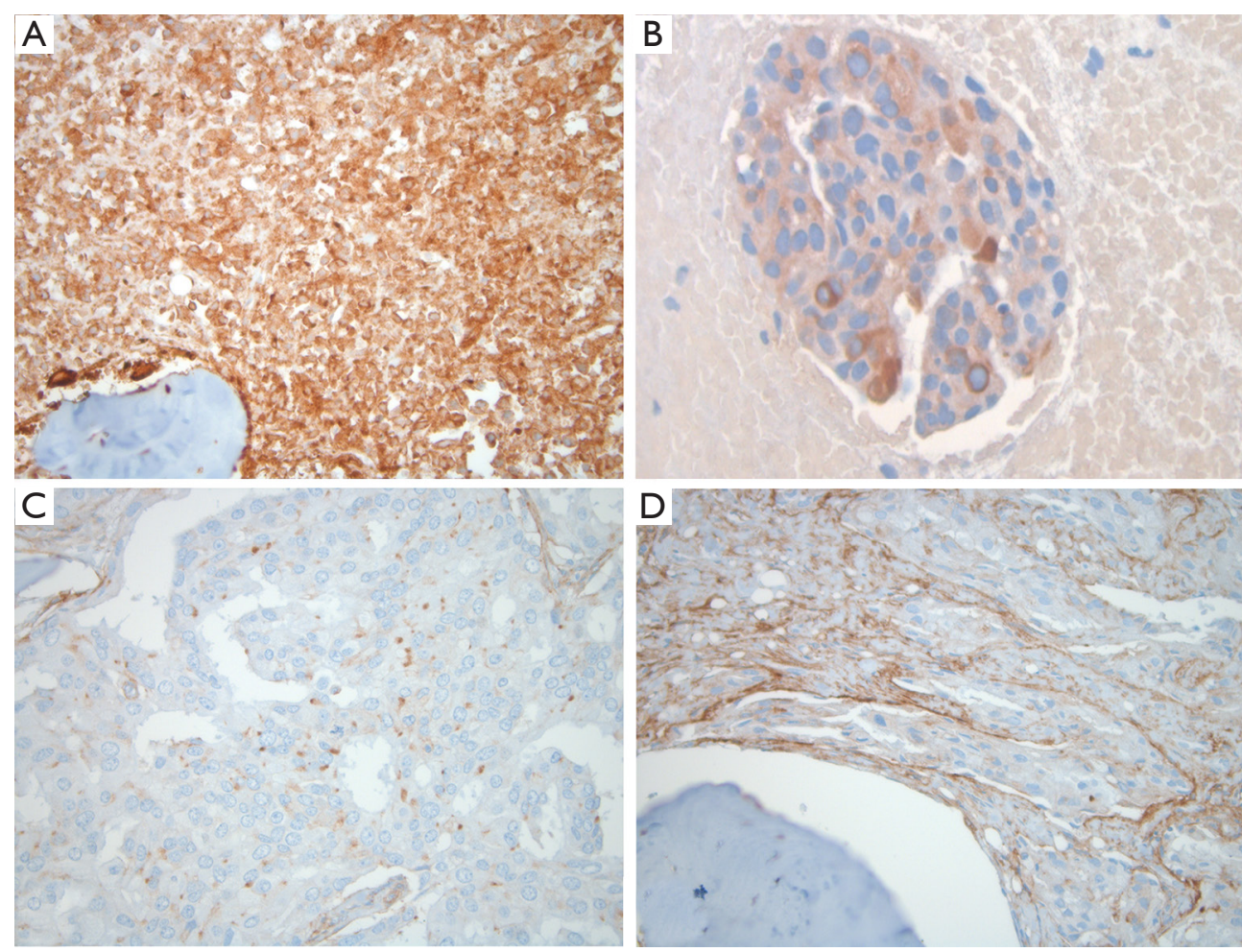

Figure 3 Prostate cancer bone metastasis samples with expression of ITGAV in a large sheet of malignant cells (A, 40×); ITGA5 in a cluster of malignant cells $(\mathrm{B}, 40 \times)$; ITGAV in a punctate cytoplasmic pattern in malignant cells $(\mathrm{C}, 40 \times)$; and ITGAV stromal expression in a rim pattern around clusters of malignant cells $(\mathrm{D}, 40 \times)$. ITGAV, integrin $\alpha \mathrm{v}$; ITGA5, integrin $\alpha 5$. 

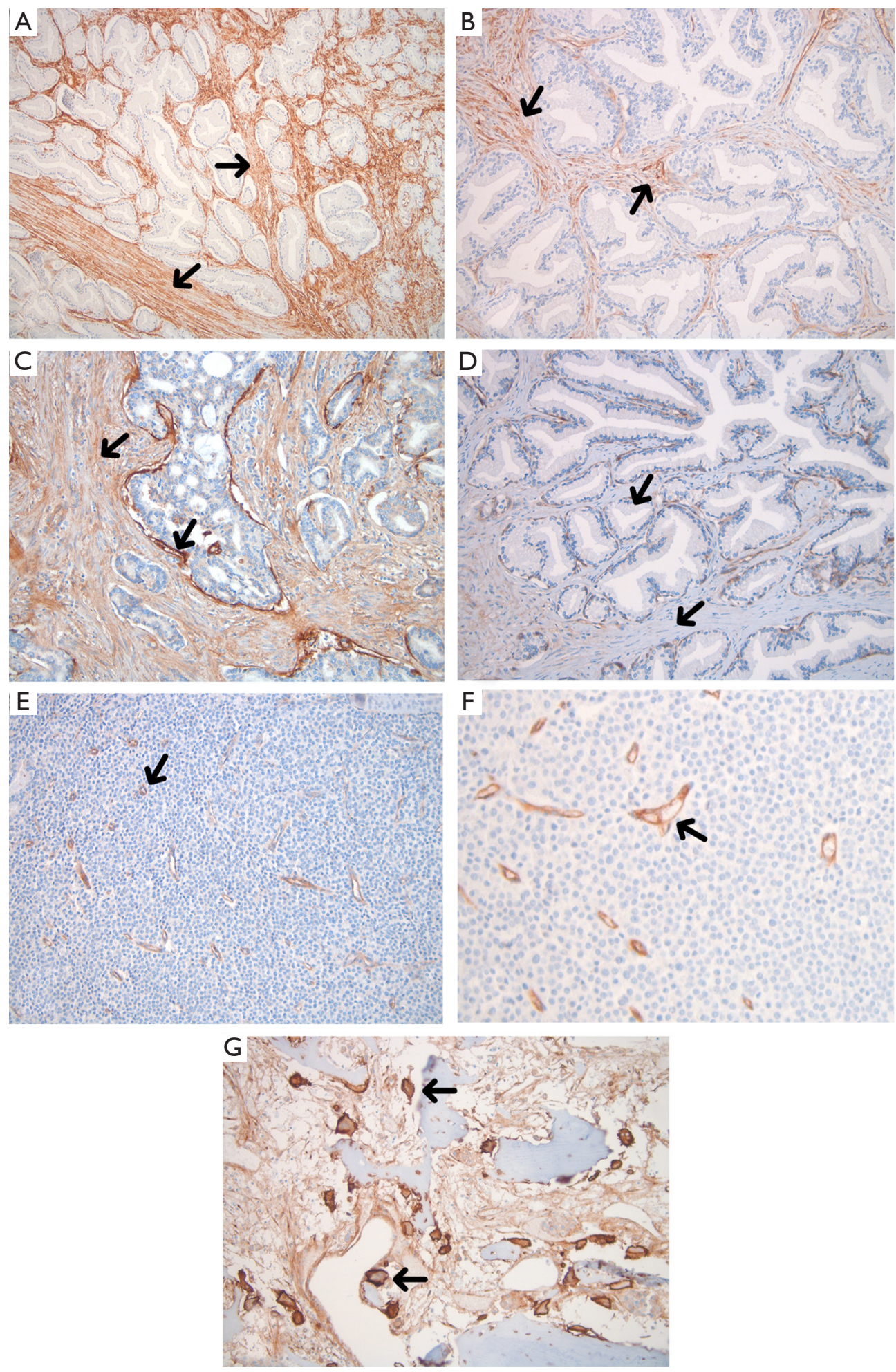

Figure 4 Integrin expression in the tumor microenvironment (20/40x): arrows indicate ITGA5 expression in the stroma adjacent to malignant (A) and benign (B) glands; ITGAV stromal expression around malignant glands, including a rim pattern (C) with negative $\alpha \mathrm{v}$ expression in the stroma around benign glands (D); ITGAV (E) and ITGA5 (F) expression in the vascular endothelium amid sheets of Gleason 5 tumor and ITGAV expression in osteoclasts from a pathologic bone sample (G). ITGAV, integrin $\alpha$; ITGA5, integrin $\alpha 5$. 


\section{Recapitulation of an ITGAV-expressing basal layer in cribriform carcinomas}

A layered expression of ITGAV was observed in primary adenocarcinomas in two specific patterns that suggest a recapitulation of the basal layer. These patterns strongly suggest a self-organizing capacity of tumors comprised of basal and luminal-differentiated components toward mirroring the hierarchical structures of the normal gland. In cribriform gland patterns which are recognized as an aggressive subset of Gleason pattern 4 histology (9), we have observed either a single basilar layer of epithelial lumenfacing cells staining specifically for ITGAV (Figure 2C) or a dense rim expression in a single stromal layer encasing cribriform glands (Figure 2D). In each case featuring strict spatial expression of ITGAV, a regulatory role for the integrin in the interface between tumor and lumen or tumor and adjacent stroma is strongly suggested.

The role of ITGAV in mediating stem-cell function in tumor cells (10) and regulating epithelial-mesenchymal transition through regulation of slug (11) has been demonstrated. For tumor-lumen interactions, it is plausible that the ITGAV expressing-layer of tumor cells is recapitulating stem-cell functions in mediating interaction with the microenvironment to organize tumor survival as well as generating differentiated ITGAV-negative tumor populations analogous to physiological luminal cells. With regard to the dense stromal rim pattern seen in cribriform glands, ITGAV has been implicated in regulating TGF-beta activation and epithelial cross-talk from cancer-associated fibroblasts (12) to support tumor survival, immune suppression and angiogenesis. Further deconvolution and validation of the biological roles of these single layers of ITGAV-expressing tumor and stromal cells will provide exciting insights into the dimensions of high-risk disease progression in prostate cancer.

\section{Membranous patterns with intense foci}

Virtually all tumors had tumor cells that expressed aberrant membranous staining of ITGAV. While these av-positive tumor cells could occur in sheets (Figure $2 A$ ), a striking exception to this pattern was intense foci ( $3+$ intensity) (Figure 2E). Here, distinct single cells and often small clusters of individual cells stained with intense membranous expression while surrounded by haloes of ITGAV-negative tumor cells. These foci resemble stem-like hubs embedded in differentiated progeny cells.

\section{Focal dense cytoplasmic pattern}

A distinct pattern of dense cytoplasmic clusters of ITGAV was noted in a subset of tumor cells. These clusters appear strongly polarized within cells suggestive of sequestration within subcellular structures. Given that integrin expression is largely regulated by trafficking (13) it is likely that endosomal pooling of these integrins is occurring, however their polarized aggregated forms are atypical for the more distributed punctate pattern associated with endosomes. Ligand-bound integrins in intracellular endosomal pools are capable of signaling function to regulate cell survival (14) and the absence of membrane expression in these tumor cells does not rule out their functional significance. Interestingly, this pattern was also identified in prostate cancer cells in the bone microenvironment.

\section{Bone-metastatic tumor cells}

To our knowledge, this study is the first to directly confirm the tissue expression of ITGAV and ITGA5 in bone metastases from prostate cancer. Study of these integrins in bone has been felt to pose methodological challenges given the acid decalcification that bone samples must undergo for processing and the damage to membrane epitopes that follows (15). A major practical limitation is the relative scarcity of such samples compared to primary tumors. However, with optimized methods described in this report, we were able to demonstrate expression of both integrins with suitable internal controls. The intense membranous and focal cytoplasmic expression patterns found in primary adenocarcinomas were represented in bone with examples of enriched expression (100\% tumor cells with $3+$ intensity) compared to primary tumors where extensive tumor expression at high-intensity was not observed.

\section{Prostate stroma and the bone microenvironment}

The tumor microenvironment in the prostate samples showed expression of ITGA5 in tumor-adjacent and normal fibroblasts in equal intensity and frequency (Figure $4 A, B$ ). ITGAV expression in stroma was variable as exemplified in the rim stromal pattern in which tumor-adjacent stroma stained strongly for ITGAV compared to benign stroma (Figure 4C,D). In Gleason 5 component, tumor-associated vascular endothelial cells were highlighted by the expression of both ITGAV and ITGA5 consistent with their known cooperative role in angiogenesis (Figure $4 E, F$ ). In bone, 
ITGAV was strongly expressed in osteoclasts associated with remodeling bone, the hallmark of prostate cancer induced osteogenesis (Figure 4G). ITGA5 was seen in endosteal cells and both integrins were also expressed in vascular endothelial cells in bone.

\section{Conclusions}

The patterns and intensity of expression of ITGAV in primary tumors together with enriched expression in bone metastases strongly supports its candidate role in the lethal bone dissemination of aggressive prostate cancer. The expression of ITGAV in cribriform carcinomas in a basilar/ luminal layer suggests that the molecular pathogenesis of these aggressive tumors may be contributed to by ITGAV. The appearance of ITGA5 specifically in bone-metastatic tumors and not in primary tumors where its expression is confined to stroma and endothelium, may be explained by bone microenvironmental-specific imprinting of integrin expression. These mechanisms may include bonemicroenvironment derived ligand-binding and outsidein signaling which upregulates epithelial expression of the integrin, induction of ITGA5 expression and function in the hypoxic environment of the bone marrow (16) and the effects of prior androgen suppression which derepresses ITGA5 expression (17). Although linked clinical histories were not accessible in this study, the surgical primary tumors were likely to be androgen-naïve and the metastatic tumors were more likely to represent castration-resistant phenotypes given the usual clinical context in which these samples are obtained. Given that ITGA5 has been demonstrated to regulate the survival threshold in prostate cancer cells (18), the bone-marrow specific upregulation of expression supports a mechanism of resistance endowed by the microenvironment.

These studies define the unique tumor-stromal expression of these fibronectin-binding integrins previously implicated in stem-cell-function, tumor cell survival and epithelial-stromal cross-talk in prostate cancer. Further studies to microdissect and validate the specific regulatory role of ITGAV in basilar/luminal patterns of expression in cribriform carcinomas of the prostate are required but are technically challenging. The findings nevertheless further advance the rationale for combined targeting of ITGAV and ITGA5 to overcome mechanisms of adaptive resistance that have been observed with single integrin targeting in prostate cancer cells. ${ }^{7}$ Patterns of expression of ITGAV in primary tumors and/or in metastatic sites with or without co- expression of ITGA5 should be annotated in translational clinical studies of combined ITGAV and ITGA5 targeting in order to enable correlation of key clinical outcomes with specific expression profiles. A combination of effective therapy deployed in biomarker-defined subpopulations of disease could together overcome the previous limitations in the field of integrin-targeting in prostate cancer $(7,19,20)$.

\section{Acknowledgments}

Frances S. Brown, MA, HT(ASCP), Tufts Cummings School of Veterinary Medicine and Discovery Pathology, Grafton MA for immunohistochemistry and Dr. Sandra Gaston, Department of Pathology and Laboratory Medicine, Tufts Medical Center for logistical management of tissue resources. These data were previously presented at the American Association of Cancer Research Annual Meeting, 2019.

Funding: The investigators gratefully acknowledge the Tufts Medical Center Prostate Cancer Research Fund: Julie and Will Person for funding support.

\section{Footnote}

Reporting Checklist: The authors have completed the STROBE reporting checklist. Available at http://dx. doi. org/10.21037/tau-19-763

Data Sharing Statement: Available at http://dx. doi. org/10.21037/tau-19-763

Conflicts of Interest: All authors have completed the ICMJE uniform disclosure form (available at http://dx. doi. org/10.21037/tau-19-763). PM reports as inventor of a pending patent assigned to Tufts Medical Center and relevant to the subject material. SN reports personal fees from Naveris, Inc., outside the submitted work. The other authors have no conflicts of interest to declare.

Ethical Statement: The authors are accountable for all aspects of the work in ensuring that questions related to the accuracy or integrity of any part of the work are appropriately investigated and resolved. All procedures performed in this study were in accordance with the Declaration of Helsinki (as revised in 2013) and approved by Tufts Medical Center Institutional Review Board (reference number 11987). Because of the retrospective nature of the research, the requirement for informed consent was waived. 
Open Access Statement: This is an Open Access article distributed in accordance with the Creative Commons Attribution-NonCommercial-NoDerivs 4.0 International License (CC BY-NC-ND 4.0), which permits the noncommercial replication and distribution of the article with the strict proviso that no changes or edits are made and the original work is properly cited (including links to both the formal publication through the relevant DOI and the license). See: https://creativecommons.org/licenses/by-nc-nd/4.0/.

\section{References}

1. Loberg RD, Logothetis CJ, Keller ET, et al. Pathogenesis and treatment of prostate cancer bone metastases: targeting the lethal phenotype. J Clin Oncol 2005;23:8232-41.

2. Visnjic D, Kalajzic Z, Rowe DW, et al. Hematopoiesis is severely altered in mice with an induced osteoblast deficiency. Blood 2004;103:3258-64.

3. Méndez-Ferrer S, Michurina TV, Ferraro F, et al. Mesenchymal and haematopoietic stem cells form a unique bone marrow niche. Nature 2010;466:829-34.

4. Shiozawa Y, Pedersen EA, Havens AM, et al. Human prostate cancer metastases target the hematopoietic stem cell niche to establish footholds in mouse bone marrow. J Clin Invest 2011;121:1298-312.

5. Joshi R, Goihberg E, Ren W, et al. Proteolytic fragments of fibronectin function as matrikines driving the chemotactic affinity of prostate cancer cells to human bone marrow mesenchymal stromal cells via the $\alpha 5 \beta 1$ integrin. Cell Adh Migr 2017;11:305-15.

6. Yang JT, Hynes RO. Fibronectin receptor functions in embryonic cells deficient in alpha 5 beta 1 integrin can be replaced by alpha $\mathrm{V}$ integrins. Mol Biol Cell 1996;7:1737-48.

7. Joshi R, Ren W, Mathew P. A bispecific antibody targeting the $\alpha \mathrm{v}$ and $\alpha 5 \beta 1$ integrins induces integrin degradation in prostate cancer cells and is superior to monospecific antibodies. Mol Cancer Res 2020;18:27-32.

8. Wolff AC, Hammond MEH, Allison KH, et al. Human epidermal growth factor receptor 2 testing in breast cancer: American Society of Clinical Oncology/College of American Pathologists Clinical Practice Guideline Focused Update. Arch Pathol Lab Med 2018;142:1364-82.

9. Kweldam CF, Wildhagen MF, Steyerberg EW, et al. Cribriform growth is highly predictive for postoperative metastasis and disease-specific death in Gleason score 7 prostate cancer. Mod Pathol 2015;28:457-64.

10. van den Hoogen C, van der Horst G, Cheung H, et al.
Integrin $\alpha v$ expression is required for the acquisition of a metastatic stem/progenitor cell phenotype in human prostate cancer. Am J Pathol 2011;179:2559-68.

11. Desgrosellier JS, Lesperance J, Seguin L, et al. Integrin alphavbeta3 drives slug activation and stemness in the pregnant and neoplastic mammary gland. Dev Cell 2014;30:295-308.

12. Khan Z, Marshall JF. The role of integrins in TGFbeta activation in the tumour stroma. Cell Tissue Res 2016;365:657-73.

13. De Franceschi N, Hamidi H, Alanko J, et al. Integrin traffic - the update. J Cell Sci 2015;128:839-52.

14. Alanko J, Ivaska J. Endosomes: emerging platforms for integrin-mediated FAK signalling. Trends Cell Biol 2016;26:391-8.

15. Heß K, Böger C, Behrens HM, et al. Correlation between the expression of integrins in prostate cancer and clinical outcome in 1284 patients. Ann Diagn Pathol 2014;18:343-50.

16. Ju JA, Godet I, Ye IC, et al. Hypoxia selectively enhances integrin $\alpha 5 \beta 1$ receptor expression in breast cancer to promote metastasis. Mol Cancer Res 2017;15:723-34.

17. Lamb LE, Zarif JC, Miranti CK. The androgen receptor induces integrin alpha6beta1 to promote prostate tumor cell survival via NF-kappaB and Bcl-xL Independently of PI3K signaling. Cancer Res 2011;71:2739-49.

18. Ren W, Joshi R, Mathew P. Synthetic lethality in PTENmutant prostate cancer is induced by combinatorial PI3K/Akt and BCL-XL inhibition. Mol Cancer Res 2016;14:1176-81.

19. Bradley DA, Daignault S, Ryan CJ, et al. Cilengitide (EMD 121974, NSC 707544) in asymptomatic metastatic castration resistant prostate cancer patients: a randomized phase II trial by the prostate cancer clinical trials consortium. Invest New Drugs 2011;29:1432-40.

20. Hussain M, Le Moulec S, Gimmi C, et al. Differential effect on bone lesions of targeting integrins: randomized phase II trial of abituzumab in patients with metastatic castration-resistant prostate cancer. Clin Cancer Res 2016;22:3192-200.

Cite this article as: Connell B, Kopach P, Ren W, Joshi R, Naber S, Zhou M, Mathew P. Aberrant integrin $\alpha \mathrm{v}$ and $\alpha 5$ expression in prostate adenocarcinomas and bone-metastases is consistent with a bone-colonizing phenotype. Transl Androl Urol 2020;9(4):1630-1638. doi: 10.21037/tau-19-763 